\title{
Hormone-Resistant Breast Carcinoma
}

National Cancer Institute

\section{Source}

National Cancer Institute. Hormone-Resistant Breast Carcinoma. NCI Thesaurus. Code C114932.

Breast carcinoma that does not respond to hormone therapy. 